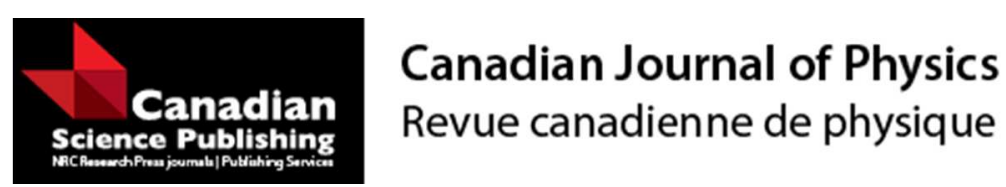

\title{
Enhancement of radiation on hydromagnetic Casson fluid flow towards a stretched cylinder with suspension of liquid- particles
}

\begin{tabular}{|r|l|}
\hline Journal: & Canadian Journal of Physics \\
\hline Manuscript ID & cjp-2017-0307.R1 \\
\hline Manuscript Type: & Article \\
\hline Date Submitted by the Author: & 05-Jun-2017 \\
\hline Complete List of Authors: & $\begin{array}{l}\text { Ramesh, G.K.; Presidency University, Bengaluru, Mathematics } \\
\text { Kumar, K.; Kuvempu University } \\
\text { Shehzad, S.A.; COMSATS Institute of Information Technology, Mathematics } \\
\text { Gireesha, B.J.; Kuvempu University }\end{array}$ \\
\hline Keyword: & $\begin{array}{l}\text { Casson fluid, cylindrical surface, convective condition, nonlinear thermal } \\
\text { radiation, liquid particle suspension }\end{array}$ \\
\hline $\begin{array}{r}\text { Is the invited manuscript for } \\
\text { consideration in a Special } \\
\text { Issue? : }\end{array}$ & N/A \\
\hline
\end{tabular}




\title{
Enhancement of radiation on hydromagnetic Casson fluid flow towards a stretched cylinder with suspension of liquid-particles
}

\author{
G.K.Ramesh ${ }^{1 *}$, K.Ganesh Kumar ${ }^{2}$, S.A.Shehzad ${ }^{3}$ and B.J.Gireesha ${ }^{2}$ \\ ${ }^{1}$ Department of Mathematics, School of Engineering, Presidency University, \\ Yelahanka, Bengaluru-560089, Karnataka, India \\ ${ }^{2}$ Department of Studies and Research in Mathematics, Kuvempu University, \\ Shankaraghatta-577 451, Shimoga, Karnataka, India. \\ ${ }^{3}$ Department of Mathematics, COMSATS Institute of Information Technology, \\ Sahiwal 57000, Pakistan \\ *Corresponding author email-id: gkrmaths@gmail.com (G.K.Ramesh) Ph: +91 9900981204

\begin{abstract}
The presence of dust particles is involved in many engineering and industrial processes like transport of petroleum, emission of smoke from vehicles, piping of power plants, treatment of waste-water and corrosive particles in mining. Due to such importance of this phenomenon, we considered the Casson liquid flow past a stretching hollow cylinder with fluidparticle suspension and convective boundary condition aspects. Numerical solution is obtained via shooting techniques after the applying of similarity variables to the governing partial equations. Key parameters of problem are discussed through graphs. It is noticed that the clean fluid temperature is always higher values as compared to dust fluid and also presence of radiation enhanced the temperature at the surface.
\end{abstract}

Keywords: Casson fluid, cylindrical surface, convective condition, nonlinear thermal radiation, liquid particle suspension.

PACS: 47.85.Dh,47.55.dd, 61.82.Bg, 67.30.eh.

\section{Introduction}

The suspension of fluid-particles has major role in nature, science and technological applications. Few examples of such applications from our daily life may include paints, food products, biological liquids, pharmaceuticals etc. Further the investigations on mechanism of fluid- 
particles submersion is a topic of hot interest nowadays because of its involvement in engineering and industry like in transport of petroleum, treatment of waste-water, emission of smoke from vehicles, piping of power plants and corrosive particles in mining. The deposition and transportation of particles is involved in different processes such as cool transport, cleaning, water and air pollution, xerography, combustion, filtration etc. [1]. Such practical implementation has engaged the researchers to elaborate the rheological features of suspension. For instance, Gireesha et al. [2] elaborated the nature of three-dimensional Couette fluid flow with suspension of liquid-particles. They computed the closed form expressions for the solution of problem. A molecular dynamic technique for the entropy generation in Couette flow with particles suspension has been described by Wang et al. [3]. Roopa et al. [4] explored the mechanism of fluid-particles submersion in time-dependent stretched surface. Manjunatha et al. [5] investigated the phenomenon of radiative heat transport in flow of dusty liquid under the aspects of heat generation. Abbas et al. [6] computed of two-phase liquid flow past through a channel. Sulochana and Sandeep [7] presented a research on the analysis of MHD viscous liquid flow with nano-particles and fluid-particles. Ramesh et al. [8-10] reported the two-phase dusty liquid flow over a permeably moving sheet under various aspects and conditions.

The consideration of boundary layer phenomenon of dusty non-Newtonian liquids with heat transport is very essential for better understanding of engineering and industrial technological problems like guided missiles rain erosion, fluidization, atmospheric fallout, luner ash flows, paint and aerosol spraying, cooling of nuclear reactor etc. Here Casson liquid model is considered as a non-Newtonian fluid. This model is very popular amongst the recent investigators. Hayat et al. [11] addressed the convective condition role in hydrodynamic Casson liquid flow. Nonlinear radiative characteristics of Casson fluid induced by a wedge have been 
numerically reported by Raju and Sandeep [12]. Hakeem et al. [13] described the features of inclined Lorentz force in Casson liquid flow induced by the moving permeable sheet. Numerical simulation for binary chemically reactive Casson liquid has been made by Abbas et al. [14]. Ramesh et al. [15] presented the numerical analysis of Casson fluid past a moving sheet with variable properties. The combined analysis of heat and mass diffusions in time-dependent Casson liquid induced by cone and flat plate has been reported by Benazir et al. [16]. They also addressed the features of double dispersion, porous medium and Joule heating in this article. Shehzad et al. [17] developed the three-dimensional flow model of Casson liquid in presence of porous media and heat generation. Rauf et al. [18] reported the numerical study to elaborate the characteristics of Robin's conditions for three-dimensional hydromagnetic Casson liquid flow past a bidirectionally moving surface. Nadeem et al. [19] provided the analytical solution for boundary layer flow of Casson fluid with magnetic field due to exponential shrinking sheet. Further Nadeem et al. [20] extended their work to three dimensional Casson fluid flows by incorporating nanoparticles.Using implicit finite-difference approximation method Iqbal et al.[21] analyzed the micopolar Casson fluid with the influence of inclined magnetic field. Mehmood et al. [22] addressed the internal heating role on Casson fluid model with microration.Impact of radiation, chemical reaction and slip effects on Casson fluid through a porous medium and addressed by Mabood et al. [23]. Singh and Dandapat [24] examined the four different types of nonlinear stretching velocities for thin film flow of Casson fluid.

Another important aspect of heat transport phenomenon which attained the special focus is called thermal radiation. The suitable knowledge of heat transfer via radiation is essential for the achievement of best quality products in industry. Several engineering processes include space vehicles, hypersonic fights, gas turbines, nuclear power plants etc. involve the phenomenon of 
radiation. Nowadays, radiative heat transport has also role in the techniques of renewable energy. Various researches [25-37] have been done in literature to describe the mechanism of radiation. Having all the above important aspects in mind, here our plan is to elaborate the features of radiation in Casson liquid flow generated by stretched cylinder through the suspension of liquidparticles. We also utilized the concept of non-linear radiation phenomenon. The Robin's condition is accounted for convective heat transport at boundary of cylinder. Numerical computation is made to find the solution of non-linear governed expressions. Various authors in the literature used different and analytical techniques to find the solutions of different fluid flow problems [38-42]. The results of dimensionless quantities have been visualized for various values of emerging physical constraints.

\section{Nomenclature}

$a \quad: \quad$ Radius of the cylinder $(m)$

\section{Greek Symbols}

$B i \quad$ : Biot number

$\beta \quad$ : Casson parameter

$B_{0} \quad$ : Magnetic field

$\beta_{1}$ : Parameter of liquid-particle interaction

$b$ : Stretching rate

$\theta$ : Dimensionless temperature

$C_{f} \quad$ : Local skin-friction coefficient

$\theta_{w}:$ Temperature heat ratio

$c_{m}:$ Specific heat of dust-particles $(J /$

$\mu \quad: \quad$ Dynamic viscosity $(\mathrm{kg} / \mathrm{ms})$ $k g k)$

$c_{p} \quad: \quad$ Specific heat of $\operatorname{liquid}(J / k g k)$

$v \quad: \quad \operatorname{Kinematic} \operatorname{viscosity}\left(\mathrm{m}^{2} / \mathrm{s}\right)$

Ec : Eckert number

$\omega \quad$ : Density ratio

$f \quad$ : Similarity variable for stream function

$\eta \quad$ : Similarity variable

$\gamma:$ Curvature parameter

$h \quad$ : Coefficient of convective heat

$\rho \quad: \quad$ Liquid density $\left(\mathrm{kg} / \mathrm{m}^{3}\right)$ transport

$K \quad$ : Stokes resistance coefficient

$k \quad:$ Thermal conductivity $(W / m K)$

$\rho_{p} \quad: \quad$ Dust-particle density $\left(\mathrm{kg} / \mathrm{m}^{3}\right)$

$\tau$ : Relaxation time of particle-phase 


\begin{tabular}{|c|c|c|c|}
\hline$l$ & : Reference length & $\tau_{T}$ & Time of thermal equilibrium \\
\hline$l^{*}$ & Dust-particles mass concentration & $\tau_{v}$ & : Dust-particle relaxation time \\
\hline$m$ & : Dust-particle mass & \multicolumn{2}{|r|}{ Subscripts } \\
\hline$M$ & Magnetic parameter & $p$ & Dust particles \\
\hline \multirow[t]{2}{*}{$N$} & Number density of the particle & $w$ & At the wall \\
\hline & phase & $\infty$ & In the free stream \\
\hline$N u_{x}$ & Local Nusselt number & & \\
\hline $\operatorname{Pr}$ & Prandtl number & & \\
\hline$q_{r}$ & Heat flux of radiation & & \\
\hline$R$ & Parameter of radiation & & \\
\hline$R e_{x}$ & Reynolds number & & \\
\hline$T_{f}$ & Hot liquid temperature $(K)$ & & \\
\hline$U_{w}$ & Stretching velocity & & \\
\hline$z, r$ & : cylindrical coordinates $(m)$ & & \\
\hline
\end{tabular}

\section{Formulation of the problem}

We propose to elaborate the laminar flow of non-Newtonian Casson fluid by stretching hollow cylinderwith suspended particles. Thez-axis is along axis of the cylinder and $r$-axis normal it. Assumed that stretching surface of the velocity $U_{w}(z)$, surface temperature $T_{w}$ and magnetic field of strength $B_{0}$ is exposed on $r$ direction. Also assumed that cylinder is heated by convection from a hot fluid at temperature $T_{f}$ itgives a heat transfer coefficient $h_{f}$. The size and shape of the dust particles is uniform and spherical. With all these assumption the governing equations are $([14],[43])$ : 


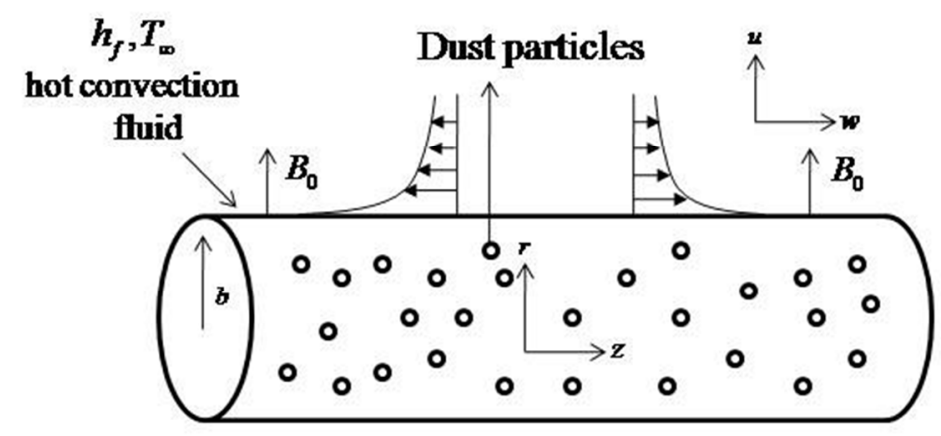

Figure 1: Geometry of problem under investigation.

$$
\begin{aligned}
& \frac{\partial(r w)}{\partial z}+\frac{\partial(r u)}{\partial z}=0 \\
& w \frac{\partial w}{\partial z}+u \frac{\partial w}{\partial r}=v\left(1+\frac{1}{\beta}\right)\left(\frac{\partial^{2} w}{\partial r^{2}}+\frac{1}{r} \frac{\partial w}{\partial r}\right)+\frac{K N}{\rho}\left(w_{p}-w\right)-\frac{\sigma B_{0}^{2} w}{\rho} \\
& w_{p} \frac{\partial w_{p}}{\partial z}+u_{p} \frac{\partial w_{p}}{\partial r}=\frac{K}{m}\left(w-w_{p}\right) \\
& w_{p} \frac{\partial u_{p}}{\partial z}+u_{p} \frac{\partial u_{p}}{\partial r}=\frac{K}{m}\left(u-u_{p}\right) \\
& \frac{\partial\left(r \rho_{p} w_{p}\right)}{\partial z}+\frac{\partial\left(r \rho_{p} u_{p}\right)}{\partial r}=0 \\
& \rho c_{p}\left(w \frac{\partial T}{\partial z}+u \frac{\partial T}{\partial r}\right)=\frac{k}{r} \frac{\partial}{\partial r}\left(r \frac{\partial T}{\partial r}\right)+\frac{N c_{p}}{\tau_{T}}\left(T_{p}-T\right)+\frac{N}{\tau_{v}}\left(w_{p}-w\right)^{2}-\frac{1}{r} \frac{\partial\left(r q_{r}\right)}{\partial r} \\
& w_{p} \frac{\partial T_{p}}{\partial z}+u_{p} \frac{\partial T_{p}}{\partial r}=-\frac{c_{p}}{\tau_{T} c_{m}}\left(T_{P}-T\right) .
\end{aligned}
$$

The boundary conditions can be defined as([5]):

$$
\begin{aligned}
& w=U_{w}(z)=b \frac{z}{l}, \quad u=0,-k \frac{\partial T}{\partial r}=h\left(T_{f}-T\right) \text { at } r=a \\
& w \rightarrow 0, w_{p} \rightarrow 0, u_{p} \rightarrow u, \rho_{p} \rightarrow \omega_{p}, T=T_{\infty}, T_{p}=T_{\infty} \text { as } r \rightarrow \infty
\end{aligned}
$$

where $(w, u, T)$ and $\left(w_{p}, u_{p}, T_{p}\right)$ are denotes components of velocity and temperature of fluid and dust particle respectively, $v$ for kinematic viscosity, $\rho$ forfluid density, $\rho_{p}$ for dust-particle density, $K$ for Stoke's constant, $m$ for thermal equilibrium at time, $N$ for number density of dust particles, $\tau_{v}$ for relaxation time of $\rho_{p}, \tau_{T}$ for thermal equilibrium time and $\omega$ for density ratio.

In the expression (6) $q_{r}$ is denoted by Rosseland approximation[43]:

$$
q_{r}=-\frac{4 \sigma^{*}}{3 k} \frac{\partial T^{4}}{\partial r}=-\frac{16 \sigma^{*}}{3 k} T^{3} \frac{\partial T}{\partial z} .
$$


Here $^{*}$ for mean absorption coefficient, $\sigma^{*}$ for Stefan-Boltzmann constant.

Dimensionless variables are defined as[5]:

$$
\begin{aligned}
& u=-\frac{a}{r} \sqrt{\frac{v b}{l}} f(\eta), \quad w=U_{w}(z) f^{\prime}(\eta), \quad \eta=\frac{r^{2}-a^{2}}{2 a} \sqrt{\frac{U_{w}}{v}}, \\
& u_{P}=\frac{a}{r} \sqrt{\frac{v b}{l}} F(\eta), \quad w_{p}=U_{w}(z) g(\eta), \quad \rho_{r}=H(\eta) \\
& T=T_{\infty}\left(1+\left(\theta_{w}-1\right) \theta\right), T_{p}=\frac{T_{p}-T_{\infty}}{T_{f}-T_{\infty}} \text {. }
\end{aligned}
$$

with $\theta_{w}=\frac{T_{f}}{T_{\infty}}, \theta_{w}>1$ being the temperature ratio parameter.

Applying equation (10) into equations (1)-(7), it reduces to the following forms

$$
\begin{aligned}
& (1+2 \eta \gamma)\left(1+\frac{1}{\beta}\right) f^{\prime \prime \prime}(\eta)+2 \gamma f^{\prime \prime}(\eta)-\left(f^{\prime}(\eta)\right)^{2}+f(\eta) f^{\prime \prime}(\eta)+ \\
& l^{*} \beta_{1} H(\eta)\left(g(\eta)-f^{\prime}(\eta)\right)-M f^{\prime}(\eta)=0 \\
& F(\eta) g^{\prime}(\eta)-g^{2}(\eta)-\beta_{1}\left(f^{\prime}(\eta)-g(\eta)\right)=0,(12) \\
& F(\eta) F^{\prime \prime}(\eta)-\left(1+\frac{\gamma}{2 \eta \gamma}\right)(F(\eta))^{2}+\beta_{1}\left[f^{\prime}(\eta)+F(\eta)\right]=0 \\
& H(\eta) g(\eta)+F(\eta) H^{\prime}(\eta)+H(\eta) F^{\prime}(\eta)-\left(1+\frac{\gamma}{2 \eta \gamma}\right) H(\eta) F(\eta)=0 \\
& (1+2 \eta \gamma)\left(1+R \frac{d}{d \eta}\left(1+\left(1+\theta_{w}\right) \theta\right)^{3}\right) \theta^{\prime \prime}(\eta)+\operatorname{Pr}\left[f(\eta) \theta(\eta)-2 f^{\prime}(\eta) \theta(\eta)\right] \\
& \quad+2 \gamma \theta^{\prime}(\eta)+\frac{N P r}{\rho \tau_{T} b}\left(\theta_{p}(\eta)-\theta(\eta)\right)+\frac{N \operatorname{PrEc}}{\rho \tau_{v}}\left[g(\eta)-f^{\prime}(\eta)\right]^{2}=0, \\
& 2 g(\eta) \theta_{p}(\eta)+F(\eta) \theta^{\prime}{ }_{p}(\eta)+\frac{c_{p} l}{\tau_{T} c_{m} b}\left(\theta_{p}(\eta)-\theta(\eta)\right)=0 .(17)
\end{aligned}
$$

The boundary conditions (9) become:

$f(\eta)=0, f^{\prime}(\eta)=1, \theta^{\prime}(0)=-B i(1-\theta(0))$ at $\eta=0$,

$f^{\prime}(\eta)=0, g(\eta)=0, F(\eta)=-f(\eta), H(\eta)=\omega, \theta_{p}(\eta)=0, \theta(\eta)=0 \quad$ as $\quad \eta \rightarrow \infty$,

$\gamma=\sqrt{\frac{l v}{a^{2}}}$ for curvature parameter, $M=\frac{\sigma B_{0}^{2} l}{\rho b}$ for magnetic parameter, $\beta_{1}=\frac{l}{\tau b}$ forliquid-particle interaction parameter, $\operatorname{Pr}=\frac{\mu c_{p}}{\rho}$ for Prandtl number, $R=\frac{16 \sigma^{*} T_{\infty}^{3}}{3 k^{*} k \rho C_{p}}$ for radiation parameter, $E c=\frac{b l^{2}}{c_{p}}$ for Eckert number, $l^{*}=\frac{m N}{\rho}$ for mass concentration dust particles and $B i=\frac{h_{f}}{k} \sqrt{\frac{v}{b}}$ Biot number. 
The skin friction coefficient and the Nusselt number canbe obtained as

$$
C_{f}=\frac{\mu\left(\frac{\partial u}{\partial r}\right)_{r=a}}{\rho U_{W}^{2}} \text { and } \quad N u_{x}=-\frac{\mu\left(\frac{\partial u}{\partial r}\right)_{r=a}}{k\left(T_{f}-T_{\infty}\right)} .
$$

Using the non-dimensional variables, we obtain

$$
C_{f} R e_{x}^{\frac{1}{2}}=\left(1+\frac{1}{\beta}\right) f^{\prime \prime}(0) \text { and } \frac{N u_{x}}{R e^{\frac{1}{2}}}=-\left[1+R \theta_{w}^{3}\right] \theta^{\prime}(0)
$$

where $R e_{x}=\frac{U_{w} x}{v}$ is the local Reynold's number.

\section{Result and Discussions}

In this section, nonlinear ordinary equations (11) to (17) along with the corresponding boundary conditions (18) do not seem feasible solution. Firstly we separated the equations (11) to (17) with conditions (18) into a set a simultaneous ten first order differential equations. To obtained solution of these equations we required corresponding initial conditions but here the values of $f^{\prime}(\eta), \theta(\eta), \theta_{p}(\eta), g(\eta)$ and $F(\eta)$ are unknown as $\eta$ tends to $\infty$, so these missing conditions can be found using the shooting method by guessing the values of $\infty$. Once we achieve the missing values called as fixed value apply the Runge-Kutta-Fehlberg integration scheme([7], [15]). The profiles of liquid velocity and liquid temperature for fluid and dust-phases are aforethought to research the influence of assorted flow dominant parameters. The numerical results of present model are matched with previous the available solutions and located to be sensible agreement as shown in table 1 . From the tables $2-5$, we can analyze the friction factor, Nusselt number, presence/absence of nonlinear thermal radiation also Newtonian and nonNewtonian behavior. The figures 2 and 3 are plotted to visualize the effects of Casson parameter $(\beta)$ on velocity distribution for both phases. Figure 2 depicts that the increase in parameter of Casson leads to reduce the curves of velocity of both phases and hence the layer of thickness decreases. On contrast, an opposite response is observed for both liquid and dust phases temperature and its relevant layer of thickness (see figure 3). 
Figures 4 and 5 describe the influences of curvature parameter $(\gamma)$ on the profiles of velocity and temperature. From figure 4,one can observe that the curves of velocity are boost up for larger parameter of curvature. Moreover the liquid velocity within the vicinity of boundary layer in is higher in the situations of cylinder in comparison to flat surface. The increment in cylinder diameter corresponds to lower profile of velocity within the layer of boundary. Figure 5 illustrate the impact of curvature parameter on temperature profile. Here the strengthening of curvature parameter sloping down the temperature profiles and corresponding thickness of layer. The behavior of the velocity for variation of magnetic parameter can be explored from figure 6 . The increasing values of parameter of magnetic lead to a lower velocity profile. The central reason for this is an applied magnetic force normal to electrically-conducting liquid which has the ability to generate drag-like force named as Lorentz force. This force acts in direction opposite to that of flow which tends to impede its motion. The dimensionless temperature of both liquid and particle-phases is remarkably boost up for larger magnetic parameter which fully agrees with physical phenomena and can be seen in figure 7.

Figure 8 is drawn to see the impact of parameter of liquid-particle interaction $\beta_{1}$ on the velocity of both phases. It is clear from figure 8 , for higher values of parameter of liquid-particle interaction lead to enhance the velocity of dust-phase while an opposite trend is obtained for velocity of liquid-phase. A friction factor is generated in liquid due to consideration of dustparticles which retards the flow. Figure 9 elucidates the effect of parameter of liquid-particle interaction on temperature profile of both liquid and particle-phases. It shows the decreasing trend over the dimensionless temperature. Figure 10 elaborates the variations of temperature for several values of Biot number. We can observe from this plot, an increase in the Biot number gives rise to the enhancement in temperature profile of both phases. This result qualitatively 
agrees with expectations, because the coefficient of heat transport enhanced for higher Biot number which is responsible for an increase in temperature.

Figures 11 exhibits the effect of Eckert number on temperature profile of the fluid and particle phase. It shows that, the increasing trend over the dimensionless temperature. The central reason for this change is that the viscosity of liquid gains energy from liquid motion and convert it into internal energy due to which liquid temperature is heated. Figure 12 is plotted to visualize the effects of radiation on temperature profile of both phases. The above graphs elucidate that, the temperature profile and corresponding thermal boundary layers of both fluid and dust phase increases by increasing the parameter of radiation. The presence of parameter of radiation provides more heat to liquid that give rise to temperature. The temperature of the fluid much more pronounced in non-linearized thermal radiation case than that of compared to linearized thermal radiation case its observed form table 2 . Figure 13 presents the impacts of parameter of temperature ratio $\left(\theta_{w}\right)$ on liquid temperature. As excepted, the increment in ratio parameter corresponds to higher temperature of both liquid and dust-phases. The ratio of momentum and thermal diffusivities is the definition of Prandtl number. The weaker thermal-diffusion is obtained in the case of higher Prandtl number that produces a reduction in temperature of both liquid and dust-phases (see figure 14).

Table 1: Comparison of the results for the dimensionless temperature gradient $\theta^{\prime}(0)$ for various values of $\operatorname{Pr}$ in the case of $\beta=0, E c=0$ and $\beta_{1}=0$.

\begin{tabular}{c|cc}
\hline \multirow{2}{*}{ Pr } & \multicolumn{2}{c}{$-\theta^{\prime}(0)$} \\
\cline { 2 - 3 } Grubka and Bobba [35] & -1.3333 & 4.7969 \\
Ali [30] & -1.3333 & 4.7969 \\
Abel et al. [37] & -1.3333 & 4.7969 \\
Present result & -1.33332 & 4.79672 \\
\hline
\end{tabular}


Table 2:Numerical values of skin friction coefficient and Nusselt number for different values physical parameters.

\begin{tabular}{|c|c|c|c|c|c|}
\hline$\gamma$ & $M$ & $\boldsymbol{\beta}$ & $\beta_{1}$ & $-\left(1+\frac{1}{\beta}\right) f^{\prime \prime}(0)$ & $-\left[1+R \theta_{w}\right] \theta^{\prime}(0)$ \\
\hline $\mathbf{0}$ & & & & 2.000003 & 1.340784 \\
\hline 0.2 & & & & 2.027095 & 1.338303 \\
\hline 0.4 & & & & 2.053544 & 1.335819 \\
\hline & 0 & & & 1.416673 & 1.338935 \\
\hline & 1 & & & 1.828354 & 1.340438 \\
\hline & 2 & a & & 2.163083 & 1.340387 \\
\hline & & 0.5 & & 2.994117 & 1.338365 \\
\hline & & 1 & & 2.448664 & 1.339959 \\
\hline & & 1.5 & & 2.237015 & 1.340373 \\
\hline & & 5 & 1 & 2.002742 & 1.340537 \\
\hline & & & 2 & 2.057506 & 1.461928 \\
\hline & & & 3 & 2.084342 & 1.518296 \\
\hline
\end{tabular}

Table 3:Numerical values of Nusselt number for different values physical parameters.

\begin{tabular}{|c|c|c|c|c|c|}
\hline$B \boldsymbol{i}$ & $E c$ & $P r$ & $\boldsymbol{R}$ & $\boldsymbol{\theta}_{w}$ & $-\left[\mathbf{1}+\boldsymbol{R} \boldsymbol{\theta}_{w}\right] \boldsymbol{\theta}^{\prime}(\mathbf{0})$ \\
\hline 1 & & & & & 1.340537 \\
\hline 2 & & & & & 2.159097 \\
\hline 3 & & & & & 2.700733 \\
\hline & 0.5 & & & & 1.340537 \\
\hline & 1 & & & & 1.248385 \\
\hline & 1.5 & & & & 1.156768 \\
\hline & & 4 & & & 1.303165 \\
\hline & & 5 & & & 1.340537 \\
\hline & & 6 & & & 1.368548 \\
\hline & & & 0 & & 0.755726 \\
\hline & & & 0.5 & & 1.340537 \\
\hline & & & 1 & & 1.879962 \\
\hline & & & & 1.1 & 1.346472 \\
\hline & & & & 1.2 & 1.340537 \\
\hline & & & & 1.3 & 1.334213 \\
\hline
\end{tabular}


Table 4:Numerical values of Nusselt number for linear and nonlinear radiation with different values physical parameters.

\begin{tabular}{|c|c|c|c|c|c|c|c|}
\hline \multirow[t]{2}{*}{$B i$} & \multirow[t]{2}{*}{$E c$} & \multirow[t]{2}{*}{$\gamma$} & \multirow[t]{2}{*}{$P r$} & \multirow[t]{2}{*}{$R$} & \multirow[t]{2}{*}{$\boldsymbol{\beta}_{1}$} & \multicolumn{2}{|c|}{$-\left[1+R \theta_{w}\right] \theta^{\prime}(0)$} \\
\hline & & & & & & Non linear radiation & Linear radiation \\
\hline 1 & & & & & & 1.340537 & 0.732318 \\
\hline 2 & & & & & & 2.159097 & 1.203488 \\
\hline 3 & & & & & & 2.700733 & 1.532061 \\
\hline & 0.5 & & & & & 1.340537 & 0.732318 \\
\hline & 1 & & $\theta$ & & & 1.248385 & 0.68163 \\
\hline & 1.5 & & & & & 1.156768 & 0.630942 \\
\hline & & 0 & 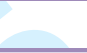 & & & 1.340784 & 0.732405 \\
\hline & & 0.2 & & & & 1.338303 & 0.730627 \\
\hline & & 0.4 & 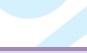 & 3 & & 1.335819 & 0.728851 \\
\hline & & & 4 & & & 1.303165 & 0.713963 \\
\hline & & & 5 & 2 & 0 & 1.340537 & 0.732318 \\
\hline & & & 6 & & & 1.368548 & 0.746081 \\
\hline & & & & 0 & 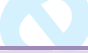 & 0.755726 & 0.755726 \\
\hline & & & & 0.5 & & 1.340537 & 0.712351 \\
\hline & & & & 1 & & 1.879962 & 0.679036 \\
\hline & & & & & 1 & 1.340537 & 0.732318 \\
\hline & & & & & 2 & 1.461928 & 0.795735 \\
\hline & & & & & 3 & 1.518296 & 0.82462 \\
\hline
\end{tabular}

Table 5: Numerical values of skin friction coefficient $\operatorname{for}(\beta=0)$ Newtonian fluid and $(\beta=1)$ nonNewtonian fluid.

\begin{tabular}{|c|cccr|}
\hline & & \multicolumn{3}{c|}{$-\left(1+\frac{1}{\beta}\right) f^{\prime \prime}(\mathbf{0})$} \\
\hline$\gamma$ & $M$ & $\beta_{1}$ & Non Newtonian fluid & Newtonian fluid \\
\hline $\mathbf{0}$ & & 2.000003 & 1.732051 \\
\hline $\mathbf{0 . 2}$ & & 2.027095 & 1.777126 \\
\hline $\mathbf{0 . 4}$ & & 2.053544 & 1.821301 \\
\hline & 0 & & 1.416673 & 1.228832 \\
\hline & & 1.828354 & 1.585583 \\
\hline & & 2.163083 & 1.875453 \\
\hline & 1 & 2.002742 & 1.736602 \\
\hline & 2 & 2.057506 & 1.784034 \\
\hline & & 2.084342 & 1.807279 \\
\hline
\end{tabular}




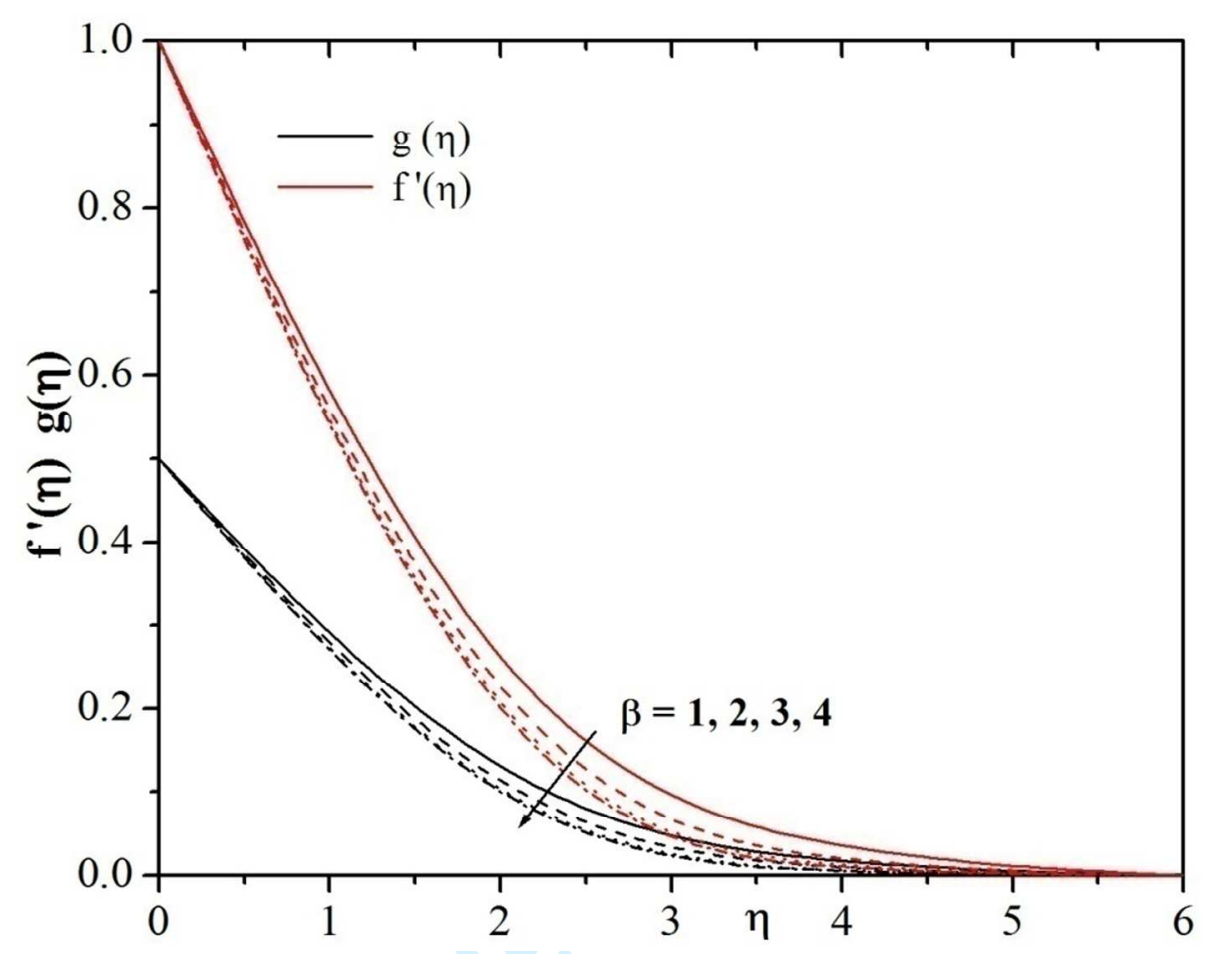

Figure 2: Velocity curves for different $\beta$.

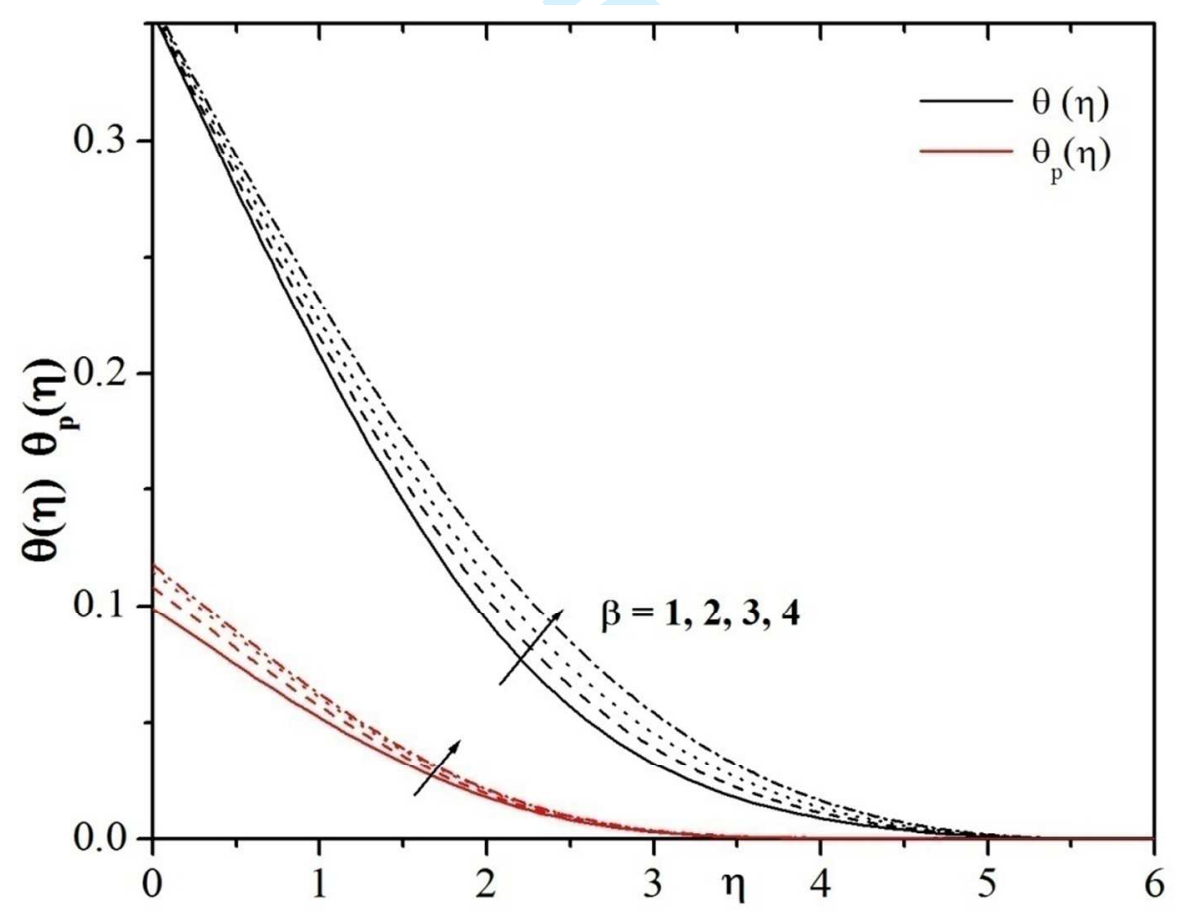

Figure 3: Temperature curves for different $\beta$. 


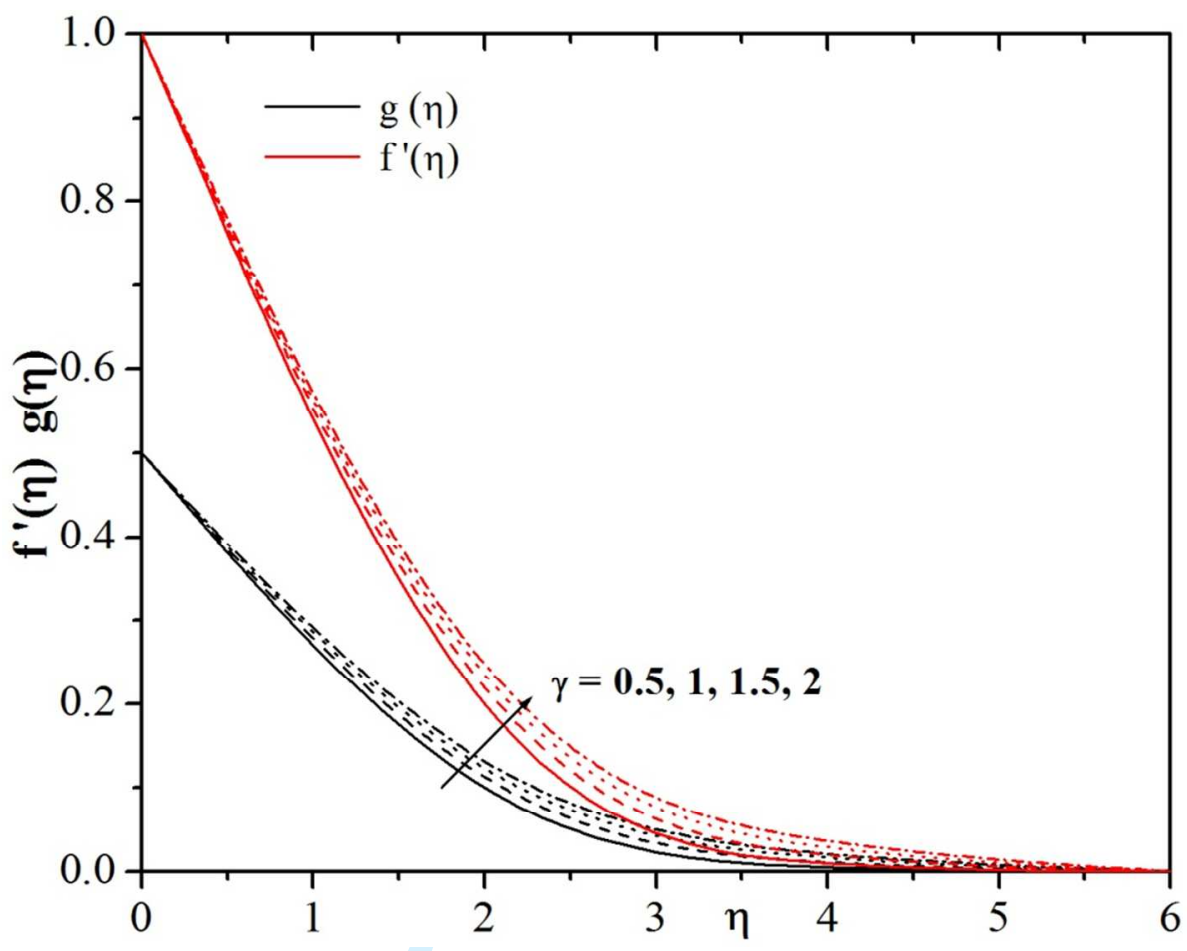

Figure 4: Velocity curves for different $\gamma$.

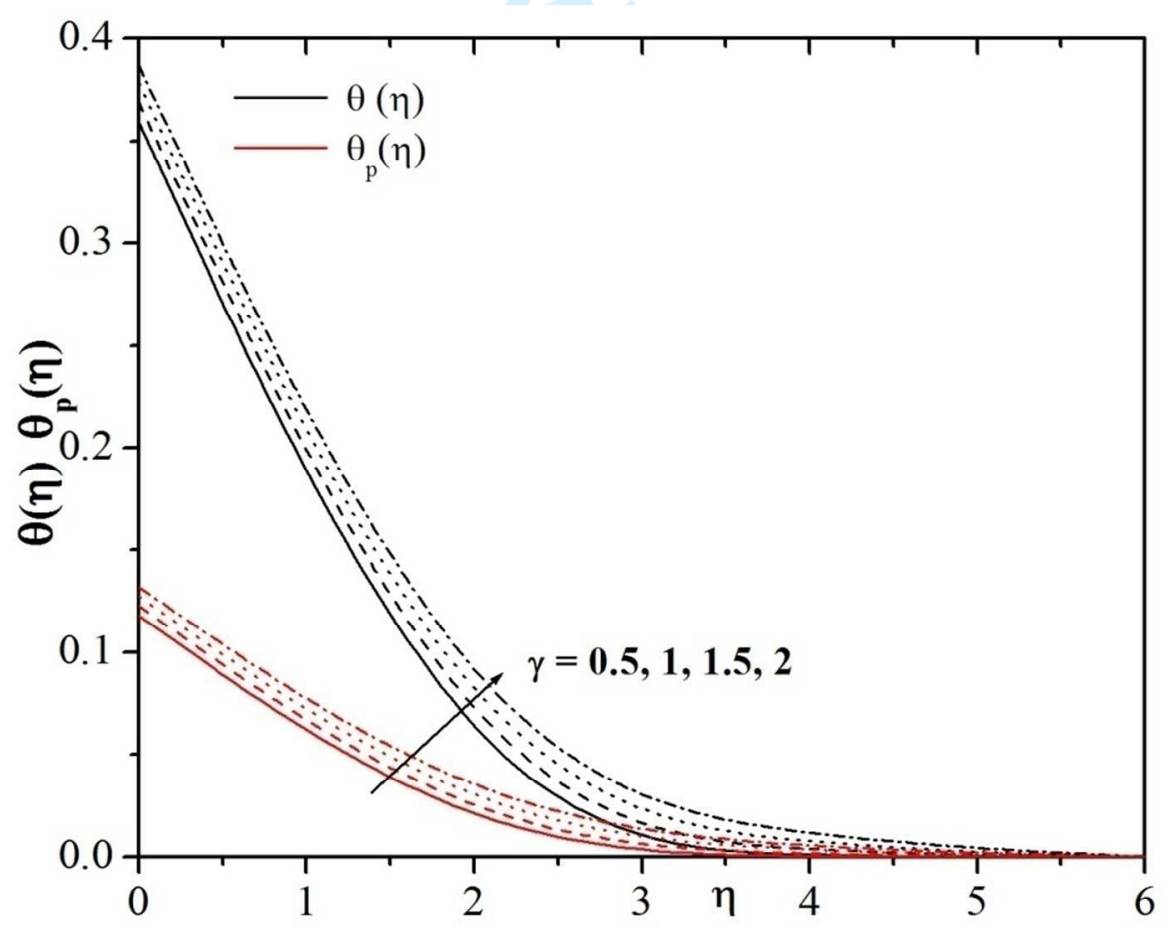

Figure 5: Temperature curves for different $\gamma$. 


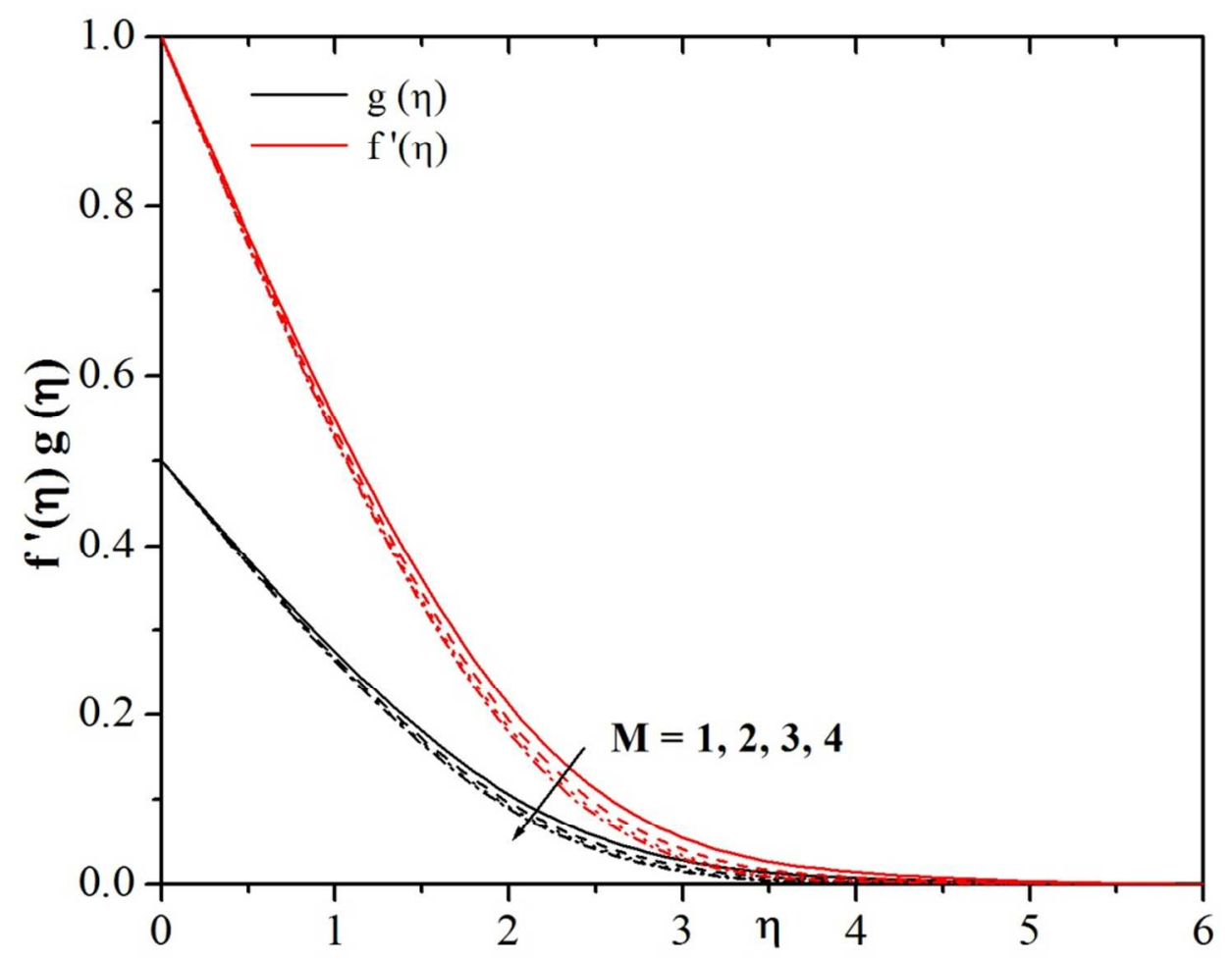

Figure 6: Velocity curves for different $M$.

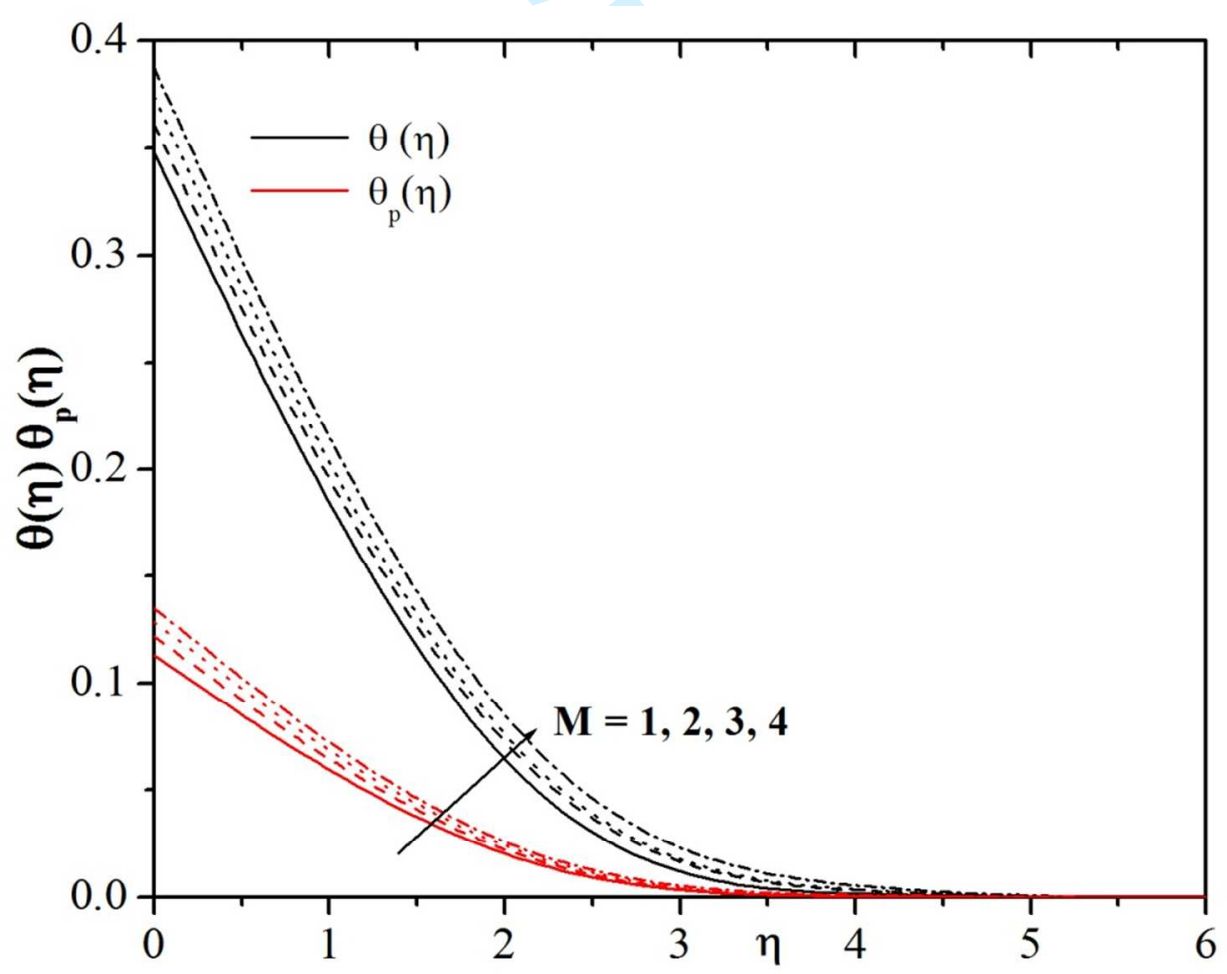

Figure 7: Temperature curves for different $M$. 


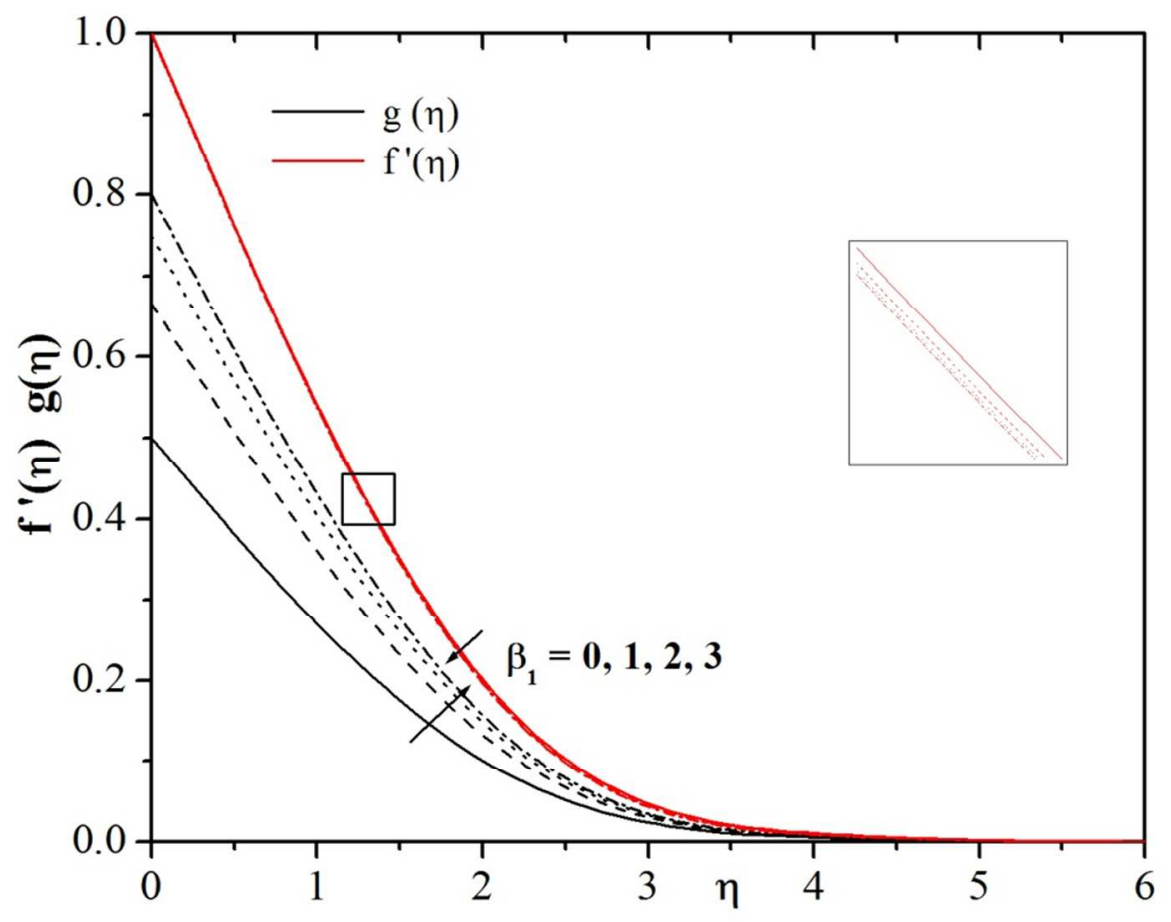

Figure 8: Velocity curves for different $\beta_{1}$.

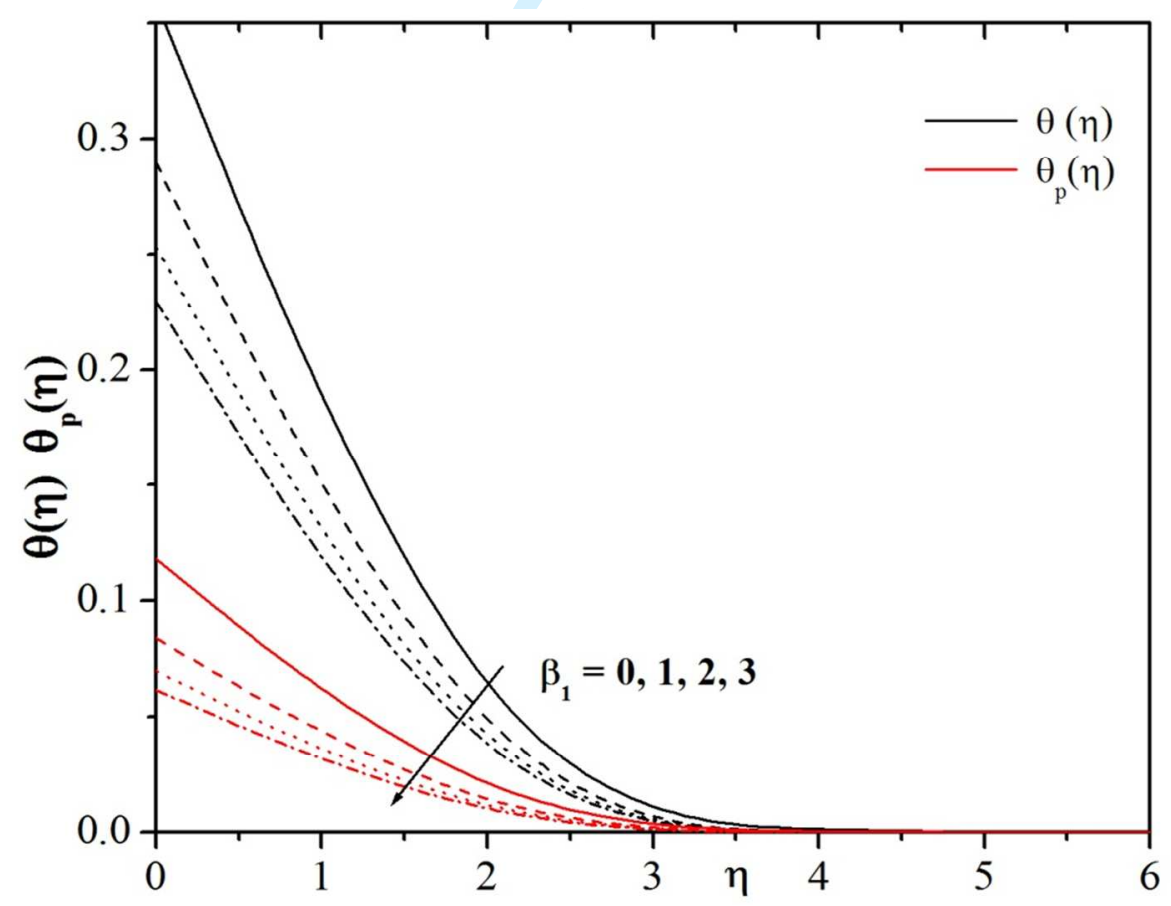

Figure 9: Temperature curves for different $\beta_{1}$. 


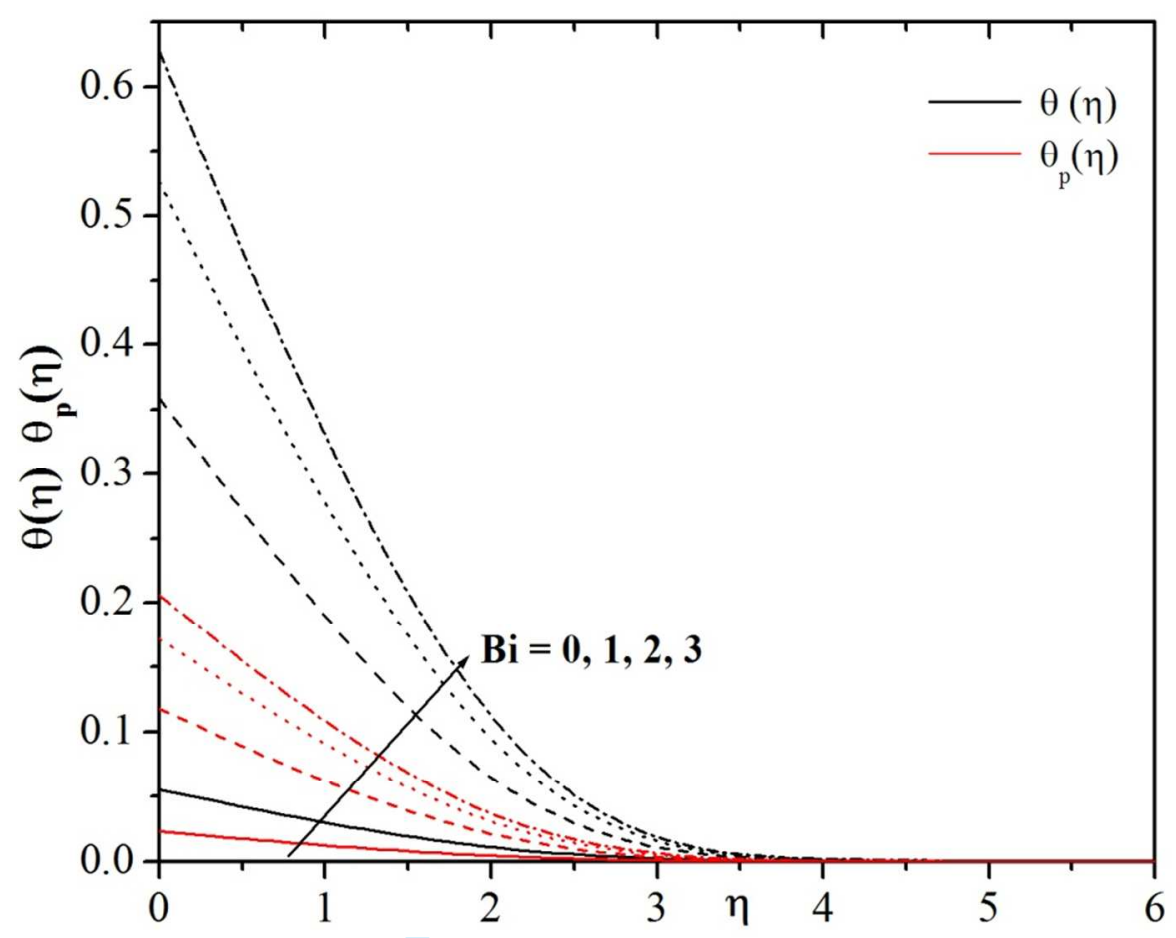

Figure 10: Temperature curves for different $B i$.

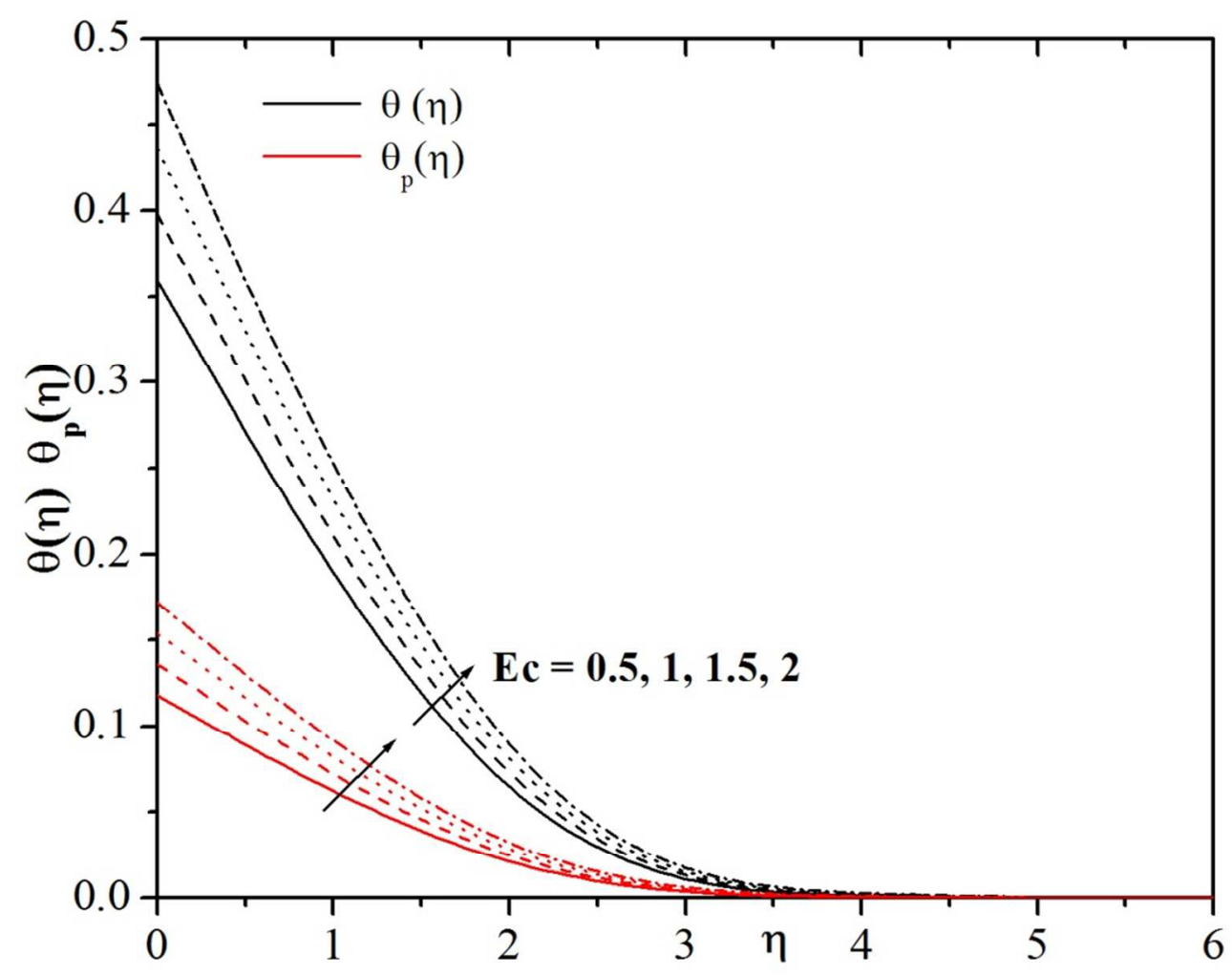

Figure 11:Temperature curves for different $E c$. 


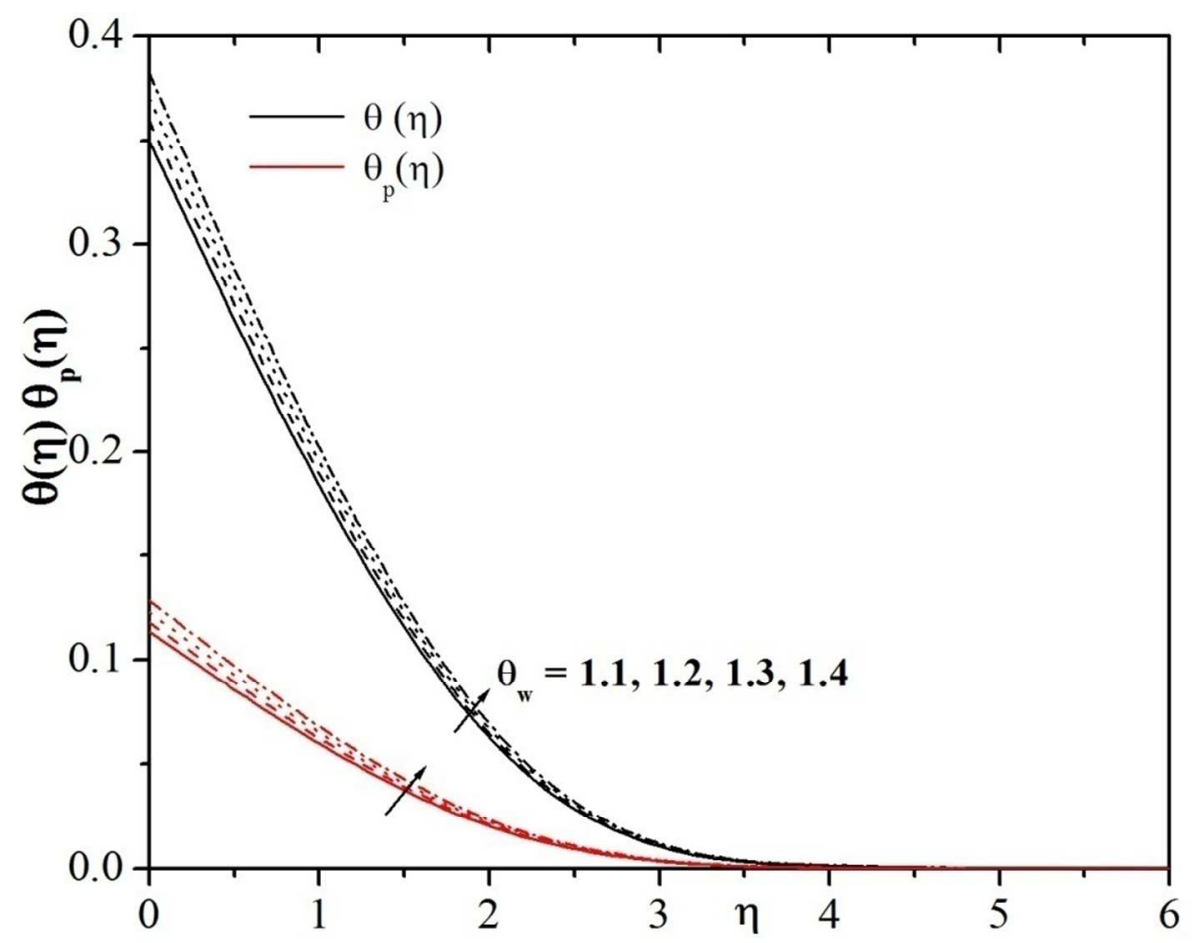

Figure 12: Temperature curves for different $\theta_{w}$.

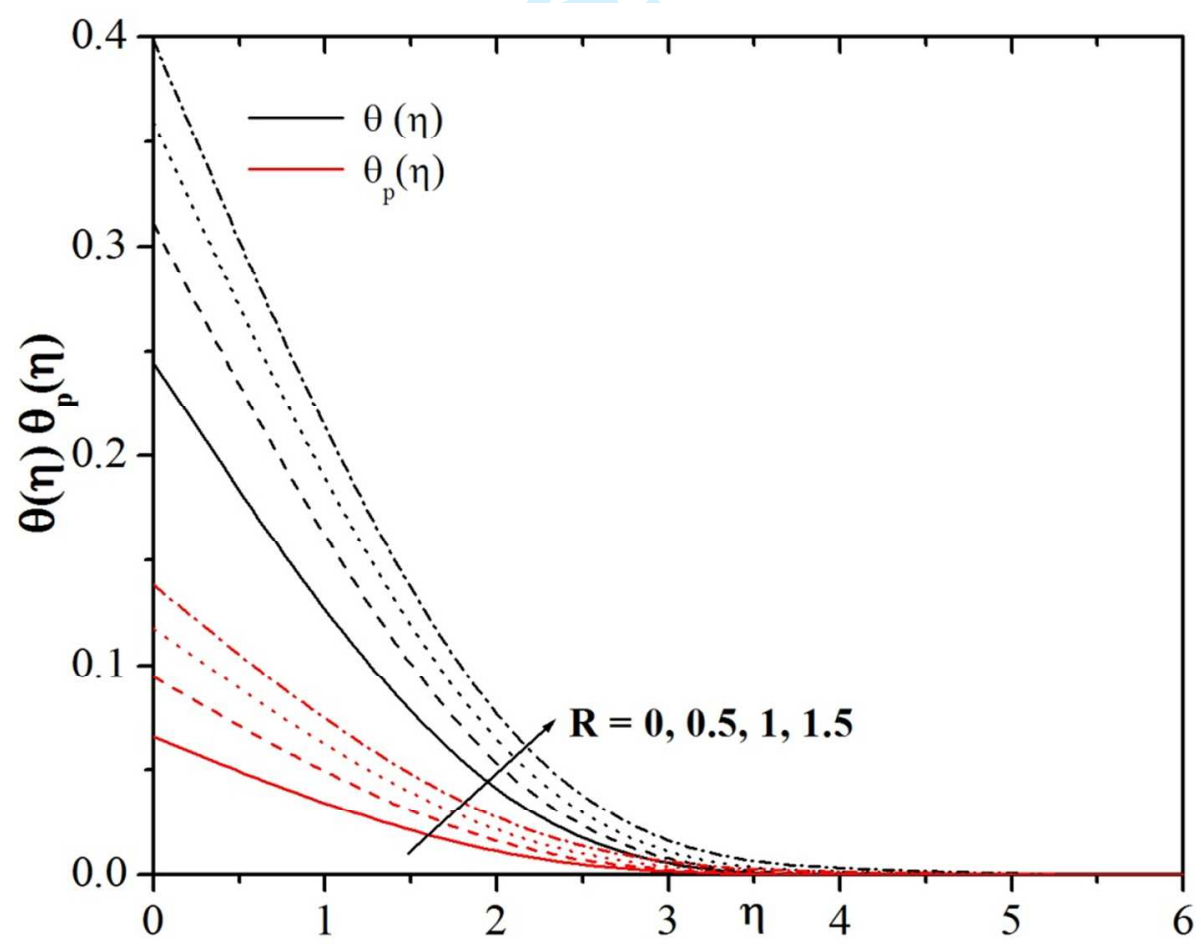

Figure 13: Temperature curves for different $R$. 


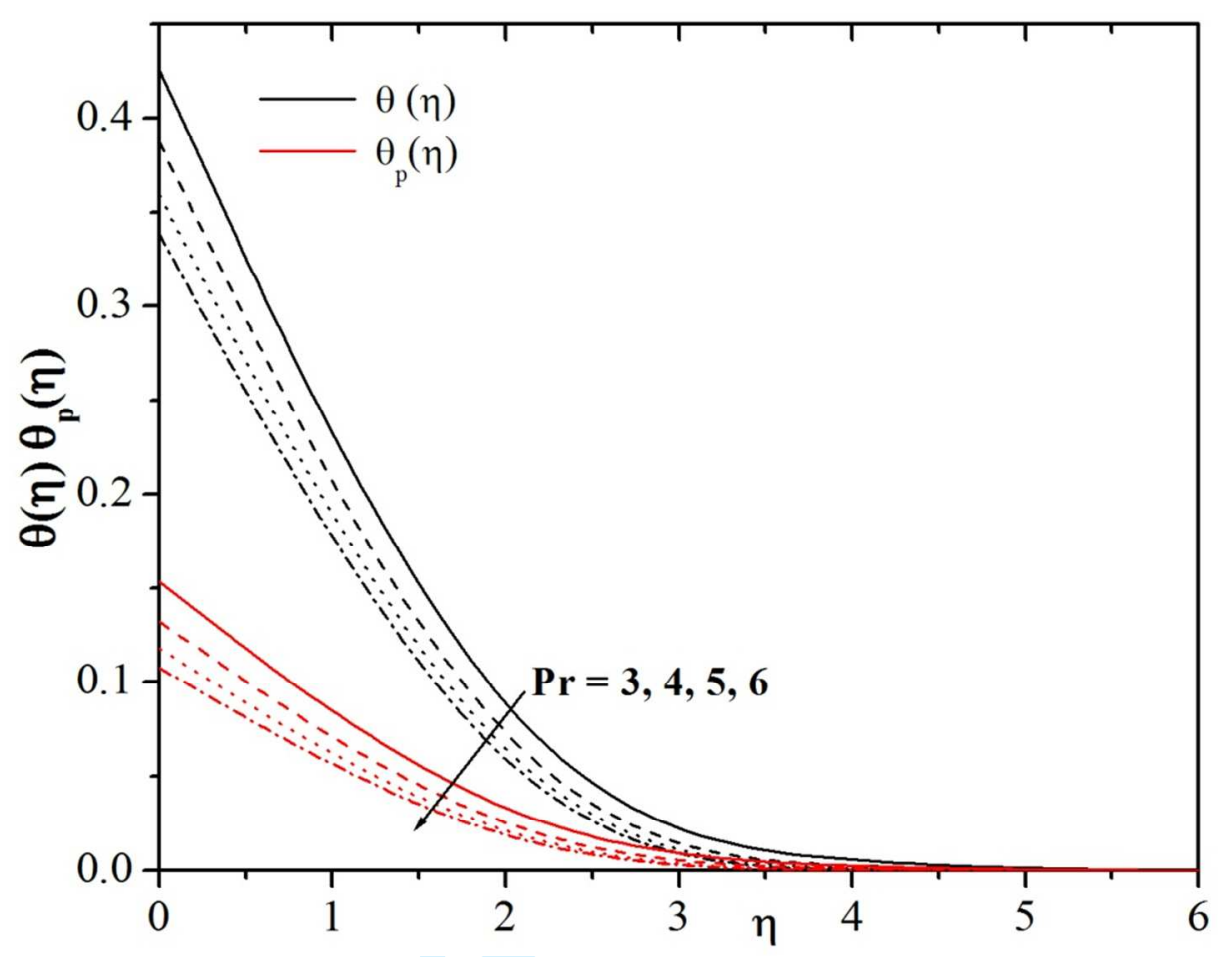

Figure 14: Temperature curves for different values $P r$.

\section{Conclusion}

This work has great importance in the field of science and technological. The considered problem is quite useful in guided missiles rain erosion, fluidization, atmospheric fallout, paint and aerosol spraying, luner ash flows and cooling of nuclear reactor. Here we explored the impact of nonlinear thermal radiation on flow and heat transfer of Casson fluid over a stretching hollow cylinder in the presence of fluid-particle suspension and convective boundary condition. Some important features regarding various physical constraints on different flow quantities of the problem are follows:

- The rising values of Casson and magnetic parameter improves the temperature profiles of the flow.

- The factor of friction reduced for larger parameter of liquid-particle interaction due to which rate of heat transport is improved. 
- The thickness of thermal layer is weaker when the Prandtl number increases. The utilization of proper Prandtl number has pivotal importance in the processes of industrial products.

- The temperature is enhanced significantly by the use of larger radiation and temperature ratio parameters.

- The fluid and dusty phase of temperature profile increase rapidly for increasing values of Biot number and Eckert number.

This work can be further extended for various models of non-Newtonian liquids like differential, integral and rate type models. Also this research can be extended for various geometries and different physical aspects. This work will serve as a basis for future research progress in field of dusty fluid.

Acknowledgments: The authors are very much thankful to the editor and referee for their encouraging comments and constructive suggestions to improve the presentation of this manuscript. Also, one of the author G.K. Ramesh expresses sincere thanks to the Management of PRESIDENCY UNIVERSITY for their kind support.

\section{Reference}

[1] M. HATAMI, KH. HOSSEINZADEH, G. DOMAIRRY, AND M.T. BEHNAMFAR, Numerical study of MHD two-phase Couette flow analysis for fluid-particle suspension between moving parallel plates, Journal of the Taiwan Institute of Chemical Engineers, Vol. 45 (2014) pp. 2238-2245.

[2] B.J. GIREESHA, A.J. CHAMKHA, C.S. VISHALAKSHI, AND C.S. BAGEWADI, Threedimensional Couette flow of a dusty fluid with heat transfer, Applied Mathematical Modelling, Vol. 36 (2012) pp.683-701.

[3] Y. WANG, Z. CHEN, AND X. LING, Entropy generation analysis of particle suspension induced by Couette flow, International Journal of Heat and Mass Transfer, Vol. 90 (2015) 
pp.499-504.

[4] G.S.ROOPA, B.J.GIREESHA, ANDC.S.BAGEWADI, Numerical investigation of mixed convection boundary layer flow of a dusty fluid over an vertical surface with radiation, AfrikaMatematika,Vol. 24 (2013) pp.487-502.

[5] P. T. MANJUNATHA.B. J. GIREESHA AND B. C. PRASANNAKUMARA, Effect of radiation on flow and heat transfer of MHD dusty fluid over a stretching cylinder embedded in a porous medium in presence of heat source, International Journal of Applied and Computational Mathematics, Vol. 3(2017) pp. 293-310.

[6] Z. ABBAS, J. HUSNAIN, ANDM. SAJID, MHD two-phase fluid flow and heat transfer with partial slip in an inclined channel, Thermal Science, Vol. 20 (2016) pp.1435-1446.

[7] C. SULOCHANA, AND N. SANDEEP, Flow and heat transfer behavior of MHD dusty nanofluid past a porous stretching/shrinking cylinder at different temperatures, Journal of Applied Fluid Mechanics, Vol. 9 (2016) pp.543-553.

[8] G.K. RAMESH, B.J. GIREESHA, AND C.S. BAGEWADI, MHD flow of a dusty fluid near the stagnation point over a permeable stretching sheet with non-uniform source/sink,International Journal of Heat and Mass Transfer, Vol. 55 (2012) pp.4900-4907.

[9] G.K. RAMESH, B.J. GIREESHA, AND R.S.R.Gorla, Boundary layer flow past a stretching sheet with fluid-particle suspension and convective boundary condition, Heat and Mass Transfer, Vol. 51 (2015) pp.1061-1066.

[10] G.K. RAMESH, AND B.J. GIREESHA, Flow over a stretching sheet in a dusty fluid with radiation effect, ASME Journal of Heat transfer, Vol. 135 (2013) pp.102702.

[11] T. HAYAT, S.A. SHEHZAD, A. ALSAEDI, AND M.S. ALHOTHUALI, Mixed convection stagnation point flow of Casson fluid with convective boundary conditions, Chinese Physics Letters, Vol. 29 (2012) pp.114704.

[12] C.S.K. RAJU, AND N. SANDEEP, Unsteady three-dimensional flow of Casson-Carreau fluids past a stretching surface, Alexandria Engineering Journal, Vol. 55 (2016) pp.1115-1126. [13] A.K.A. HAKEEM, P. RENUKA, N.V. GANESH,R. KALAIVANAN, ANDB. GANGA, Influence of inclined Lorentz forces on boundary layer flow of Casson fluid over an impermeable stretching sheet with heat transfer, Journal of Magnetism and Magnetic Materials, Vol. 401 (2016) pp.354-361.

[14] Z. ABBAS, M. SHEIKH, AND S.S. MOTSA, Numerical solution of binary chemical 
reaction on stagnation point flow of Casson fluid over a stretching/shrinking sheet with thermal radiation, Energy, Vol. 95 (2016) pp.12-20.

[15] G.K. RAMESH, B.C. PRASANNAKUMARA, B.J. GIREESHA, AND M M. RASHIDI, Casson fluid flow near the stagnation point over a stretching sheet with variable thickness and Radiation, Journal of Applied Fluid Mechanics, Vol. 9 (2016) pp.1115-1122.

[16] A.J. BENAZIR, R. SIVARAJ, AND M. M. RASHIDI, Comparison between Casson fluid flow in the presence of heat and mass transfer from a vertical cone and flat plate, J. Heat Transfer, Vol. 138 (2016) pp.112005.

[17] S.A. SHEHZAD, T. HAYAT, ANDA. ALSAEDI, Three-dimensional MHD flow of Casson fluid in porous medium with heat generation, Journal of Applied Fluid Mechanics, Vol. 9 (2016) pp. 215-223.

[18] A. RAUF, M.K. SIDDIQ, F.M. ABBASI, M.A. MERAJ, M. ASHRAF, ANDS.A. SHEHZAD, Influence of convective conditions on three dimensional mixed convective hydromagnetic boundary layer flow of Casson nanofluid, Journal of Magnetism and Magnetic Materials, Vol. 416 (2016) pp.200-207.

[19] S. NADEEM, R. U1 HAQ AND C. LEE, MHD flow of a Casson fluid over an exponentially shrinking sheet, Scientia Iranica, Vol. 19(2012) pp. 1550-1553.

[20] S. NADEEM, R. U1 HAQ, AND N. S. AKBAR,MHD three-dimensional boundary layer flow of Casson nanofluid past a linearly stretching sheet with convective boundary condition, IEEE Transactions on Nanotechnology, Vol. 13(2014) pp. 109 - 115

[21] Z. IQBAL, R. MEHMOOD, E. AZHA, AND Z. MEHMOOD,Impact of inclined magnetic field on micropolar Casson fluid using Keller box algorithm, European Physical Journal Plus, Vol. 132(2017) pp. 175.

[22]Z. MEHMOOD, R. MEHMOOD, AND Z. IQBAL, Numerical investigation of micropolar Casson fluid over a stretching sheet with internal heating, Communications in Theoretical Physics, Vol. 67(2017) pp. 443.

[23]F. MABOOD, W.A. KHAN, AND A.I.M. ISMAIL,Multiple slips effects on MHD Casson fluid flow in porous media with radiation and chemical reaction, Canadian Journal of Physics, Vol. 94(2016) pp. 26-34.

[24] S.K. SINGH AND B.S. DANDAPAT, Thin film flow of Casson liquid over a nonlinear stretching sheet in the presence of a uniform transverse magnetic field, Canadian Journal of 
Physics, Vol. 93 (2015) pp. 1067-1075

[25] K. HSIAO, Energy conversion conjugate conduction--convection and radiation over nonlinearly extrusion stretching sheet with physical multimedia effects, Energy, Vol. 59 (2013) pp.494-502.

[26] L. ZHENG, C. ZHANG, X. ZHANG, ANDJ. ZHANG, Flow and radiation heat transfer of a nanofluid over a stretching sheet with velocity slip and temperature jump in porous medium, Journal of the Franklin Institute, Vol. 350 (2013) pp.990-1007.

[27] M. TURKYILMAZOGLU, ANDI. POP, Heat and mass transfer of unsteady natural convection flow of some nanofluids past a vertical infinite flat plate with radiation effect, International Journal of Heat and Mass Transfer, Vol. 59 (2013) pp.167-171.

[28] K. HSIAO, Nanofluid flow with multimedia physical features for conjugate mixed convection and radiation, Computers \& Fluids, Vol. 104 (2014) pp.1-8.

[29] J. LI, L. LIU, L. ZHENG, ANDB. BIN-MOHSIN, Unsteady MHD flow and radiation heat transfer of nanofluid in a finite thin film with heat generation and thermophoresis, Journal of the Taiwan Institute of Chemical Engineers, Vol. 67 (2016) pp.226-234.

[30] M. SHEIKHOLESLAMI, AND S.A. SHEHZAD, Thermal radiation of ferrofluid in existence of Lorentz forces considering variable viscosity, International Journal of Heat and Mass Transfer,Vol. 109 (2017) pp.82-92.

[31] M. SHEIKHOLESLAMI, ANDM. SHAMLOOEI, $\mathrm{Fe}_{3} \mathrm{O}_{4}-\mathrm{H}_{2} \mathrm{O}$ nanofluid natural convection in presence of thermal radiation, International Journal of Hydrogen Energy, Vol. 42 (2017) pp.5708-5718.

[32] I.L. ANIMASAUN, C.S.K. RAJU, ANDN. SANDEEP, Unequal diffusivities case of homogeneous--heterogeneous reactions within viscoelastic fluid flow in the presence of induced magnetic-field and nonlinear thermal radiation, Alexandria Engineering Journal, Vol.55 (2016) pp.1595-1606.

[33] T. HAYAT, S. QAYUM, A. ALSAEDI, AND S.A. SHEHZAD, Nonlinear thermal radiation aspects in stagnation point flow of tangent hyperbolic nanofluid with double diffusive convection, Journal of Molecular Liquids, Vol. 223 (2016) pp.969-978.

[34] G.K. RAMESH, B.C. PRASANNAKUMARA, B.J. GIREESHA, S.A. SHEHZAD, ANDF.M. ABBASI, Three dimensional flow of Maxwell fluid with suspended nanoparticles past a bidirectional porous stretching surface with thermal radiation, Thermal Science and 
Engineering Progress, Vol. 1 (2017) pp.6-14.

[35]L.J. GRUBKA, AND K.M. BOBBA, Heat Transfer characteristics of a continuous stretching surface with variable temperature. ASME Journal of Heat Transfer, Vol. 107(1985) pp.248-250.

[36] M.E. ALI, Heat transfer characteristics of a continuous stretching surface. Warme andStofffibertragung, Vol. 29 (1994) pp. 227-234.

[37] M.S. ABEL, E. SANJAYANAND, ANDM.M. NANDEPPANAVAR, Viscoelastic MHD flow and heat transfer over a stretching sheet with viscous and ohmic dissipation. Commuicationsin Nonlinear Science and Numerical Simulation, Vol. 13(2008)pp. 1808-1821.

[38] S. NADEEM, R. MMEHMOOD, AND N.S. AKBAR, Optimized analytical solution for oblique flow of a Casson-nano fluid with convective boundary conditions.International Journal of Thermal Sciences, Vol. 78 (2014)pp. 90-100.

[39] S. RANA, R. MMEHMOOD, N.S. AKBAR, Mixed convective oblique flow of a Casson fluid with partial slip, internal heating and homogeneous-heterogeneous reactions.Journal of Molecular Liquids, Vol. 222 (2016)pp. 1010-1019.

[40] S. NADEEM, S. MASOOD, R. MEHMOOD,AND M.A. SADIQ, Optimal and numerical solutions for an MHD micropolar nanofluid between rotating horizontal parallel plates.Plos One, Vol. 10 (2015) pp. e0124016.

[41] R. MEHMOOD, S. NADEEM, ANDN. AKBAR, Oblique stagnation flow of Jeffery fluid over a stretching convective surface: Optimal solution.International Journal of Numerical Methods for Heat \&Fluid Flow, Vol. 25 (2015) pp. 454-471.

[42] S. NADEEM, R. MEHMOOD, ANDS.S. MOTSA, Numerical investigation on MHD oblique flow of Walter's B type nano fluid over a convective surface.InternationalJournal ofThermal Sciences, Vol. 92 (2015) pp. 162-172.

[43] A. RASEKH, D. D. GANJI, S. TAVAKOLI, , H. EHSANI, ANDS. NAEEJEE, MHD flow and heat transfer of a dusty fluid over a stretching hollow cylinder with a convective boundary condition. Heat Transfer Asian Research, Vol. 43 (2014) pp. 221-232. 